\title{
Zwei aussichtsreiche Substanzen bei diastolischer Herzschwäche
}

\section{Zwei beim europäischen Kardiolo- genkongress vorgestellte Studien versprechen neue Therapieoptionen bei diastolischer Herzinsuffizienz. Die Hoffnungsträger: Ein Aldosteron- antagonist und ein Angiotensin- Rezeptor-Neprilysin-Inhibitor.}

- Mehr als 50\% aller Patienten mit Herzinsuffizienz weisen keine Pumpschwäche, sondern eine diastolischen Compliancestörung auf, häufig auf dem Boden einer Hypertonie. Die Lebenserwartung dieser Patienten ist reduziert, evidenzbasierte Therapien sind rar.

\section{Die ALDO-DHF-Studie mit Spironolacton}

Aldosteron kann in der Pathogenese eine Rolle spielen, denn das Steroidhormon fördert Fibrose und Hypertrophie des Myokards sowie die Versteifung der Gefäße. In der doppelblinden ALDODHF-Studie wurde jetzt der Nutzen einer Spironolacton-Therapie bei $422 \mathrm{~Pa}-$ tienten in frühen Stadien der diastolischen Herzschwäche untersucht.

Wie Prof. Burkert Pieske von der Universitätsklinik in Graz berichtete, verbesserte Spironolacton $(25 \mathrm{mg} / \mathrm{d})$ eine Reihe von Parametern der diastolischen Herzfunktion sowie der Herzstruktur, unabhängig von der gleichzeitigen Blutdrucksenkung. Auf Belastbarkeit, Herzinsuffizienz-Stadium oder Lebensqualität hatte die Behandlung keinen Einfluss.

Im nächsten Jahr werden die Ergebnisse der TOPCAT-Studie erwartet, die die langfristige Wirksamkeit des Aldosteronblockers auf klinische Endpunkte bei über 3000 Patienten untersucht.

\section{Die PARAMOUNT-Studie mit einem} Inhibitor von $\mathrm{AT}_{1}$ und Neprilysin Eine weiterer Ansatzpunkt könnte die bei diastolischer Relaxationsstörung verminderte Aktivität des natriuretischen Systems sein. In der sog. PARAMOUNT-Studie wurde die Wirksamkeit von LCZ696 bei 308 Patienten mit Herzinsuffizienz und erhaltener Ejektionsfraktion (NT pro-BNP-Werte $>400 \mathrm{pg} /$ $\mathrm{ml}$ ) getestet. LCZ696 hat einen dualen Wirkmechanismus: Es steigert die Aktivität von natriuretischem Peptid und enthält zusätzlich Valsartan, blockt somit den Angiotensin-1-Rezeptor.

Im Vergleich zu einer alleinigen Valsartan-Gabe reduzierte LCZ696 das NT pro-BNP (primärer Endpunkt) nach zwölf Wochen um 23\% ( $p=0,005)$, berichtete Prof. Scott Solomon, Harvard Medical School in Boston. Gleichzeitig wurde das Volumen des linken Vorhofes reduziert. Beide Parameter sind mit der Prognose dieser Krankheit assoziiert, so Solomon. Überdies zeigten sich Parameter der diastolischen Herzfunktion verbessert. Auch die NYHA-Klasse verbesserte sich. Die Verträglichkeit war gut.

Monotherapien mit ACE-Hemmern oder $\mathrm{AT}_{1}$-Blockern hatten in größeren Studien bei diastolischer Herzinsuffizienz bisher überwiegend nur einen Trend zu einer klinischen Verbesserung ergeben. Die Autoren empfehlen jetzt große Endpunktstudien mit LCZ696. Solche laufen bereits bei systolischer Herzinsuffizienz und bei Hypertonie.

DR. MED. DIRK EINECKE .

- Kongress der European Society of Cardiology 2012, München, August 2012

\section{ST-Hebungs-Myokardinfarkt}

\section{Rauchverbot kommt vor allem Nichtrauchern zugute}

\section{Das Nichtraucherschutzgesetz wird seinem Namen gerecht: Vor allem bei Nichtrauchern trägt es zum Rück- gang der Infarkthäufigkeit bei.}

- Das Nichtraucherschutzgesetz (NRSG) von 2007 hat das Rauchen in öffentlichen Gebäuden und in der Gastronomie bis auf wenige Ausnahmen verboten. Der kürzlich vorgestellte Jahresbericht des Deutschen Krebsforschungszentrums zeigt aber, dass die Kontrollen in einigen Bundesländern wohl strenger werden müssen, etwa in Bremen.

Dennoch scheint das Gesetz auch in Bremen Wirkung zu zeigen, wie eine Auswertung des Bremer STEMI-Registers zeigt. Die Zahl der monatlichen Einweisungen wegen transmuraler Herzinfarkte ging nach Inkrafttreten des NRSG von 65 auf 55 zurück - ein Rückgang um 16\%, berichtete J. Schmucker, Klinikum Links der Weser beim europäischen Herzkongress.

Interessant war eine Subgruppen-Analyse: Die Infarkthäufigkeit war nämlich nur bei Nichtrauchern signifikant rückläufig (um 26\%), und hier insbesondere bei jüngeren Nichtrauchern unter 65 Jahren (um 32\%). Die Zahl der aktiv rauchenden STEMI-Patienten war hingegen etwas angestiegen.

Nach Ansicht der Bremer Mediziner lassen diese Ergebnisse vermuten, dass das NRSG insbesondere Nichtraucher vor den schädlichen Folgen des Passivrauchens schützt.

Peter Overbeck .

- J. Schmucker, et al.; Smoking ban and incidence of STEMI: non-smokers benefit most? Abstract P3541, ESC-Kongress München 2012 